\title{
Wireless Mesh Networks and Cloud Computing for Real Time Environmental Simulations
}

Kropf, Peter ; Schiller, Eryk ; Brunner, Philip ; Schilling, Oliver ; Hunkeler, Daniel ; Lapin, Andrei

\begin{abstract}
Predicting the influence of drinking water pumping on stream and groundwater levels is essential for sustainable water management. Given the highly dynamic nature of such systems any quantitative analysis must be based on robust and reliable modeling and simulation approaches. The paper presents a wireless mesh-network framework for environmental real time monitoring integrated with a cloud computing environment to execute the hydrogeological simulation model. The simulation results can then be used to sustainably control the pumping stations. The use case of the Emmental catchment and pumping location illustrates the feasibility and effectiveness of our approach even in harsh environmental conditions.
\end{abstract}

DOI: https://doi.org/10.1007/978-3-319-06538-0_1

Posted at the Zurich Open Repository and Archive, University of Zurich

ZORA URL: https://doi.org/10.5167/uzh-175021

Book Section

Accepted Version

Originally published at:

Kropf, Peter; Schiller, Eryk; Brunner, Philip; Schilling, Oliver; Hunkeler, Daniel; Lapin, Andrei (2014). Wireless Mesh Networks and Cloud Computing for Real Time Environmental Simulations. In: Boonkrong, Sirapat; Unger, Herwig; Meesad, Phayung. Recent Advances in Information and Communication Technology. Cham: Springer, 1-11.

DOI: https://doi.org/10.1007/978-3-319-06538-0_1 


\title{
Wireless Mesh Networks and Cloud Computing for Real Time Environmental Simulations
}

\author{
Peter Kropf ${ }^{1}$, Eryk Schiller ${ }^{1}$, Philip Brunner ${ }^{2}$, Oliver Schilling ${ }^{2}$, Daniel \\ Hunkeler $^{2}$, and Andrei Lapin ${ }^{1}$ \\ 1 Université de Neuchâtel, Computer Science department (IIUN), \\ CH-2000 Neuchâtel, Switzerland, \\ peter.kropf , eryk.schiller, andrei.lapin@unine.ch \\ 2 Université de Neuchâtel, Centre for Hydrogeology and Geothermics (CHYN), \\ CH-2000 Neuchâtel, Switzerland, \\ philip.brunner, oliver.schilling, daniel hunkeler@unine.ch
}

\begin{abstract}
Predicting the influence of drinking water pumping on stream and groundwater levels is essential for sustainable water management. Given the highly dynamic nature of such systems any quantitative analysis must be based on robust and reliable modeling and simulation approaches. The paper presents a wireless mesh-network framework for environmental real time monitoring integrated with a cloud computing environment to execute the hydrogeological simulation model. The simulation results can then be used to sustainably control the pumping stations. The use case of the Emmental catchment and pumping location illustrates the feasibility and effectiveness of our approach even in harsh environmental conditions.
\end{abstract}

Keywords: wireless mesh network, cloud computing, data assimilation, environmental measurements, hydrogeological modelling and simulation, ground water abstraction

\section{Introduction}

Climatic or hydrological systems are driven by highly dynamic forcing functions. Quantitative numerical frameworks such as simulation models are powerful tools to understand how these functions control the systems' response. Models are, however, always imperfect descriptions of reality and therefore model calculations increasingly deviate from the "real" physical conditions of the environmental system simulated. We can alleviate these biases by a real time integration of field data into the modeling framework (data assimilation). To accomplish this goal, we have to constantly monitor the environment through dense networks of sensors deployed over the geographical area concerned. The technology should provide high performance even in the case of harsh meteorological conditions (snow, low temperatures, fog, strong winds, etc.) and other location and infrastructure related limitations like high altitude, lack of access to the power grid, and limited accessibility (resulting in long access delays inducing significant installation/maintenance costs). 


\subsection{Wireless Infrastructure}

In principle, communication networks can be wired or wireless. However, building a vast and complex wired infrastructure is costly and can be technically impossible in remote locations. An alternative are radio-based technologies, which do not require expensive cabled infrastructures. Moreover, this technological choice is extremely portable, because one can easily transfer equipment from one location to another when necessary. The first choice transport technology would be GSM/UMTS, however, this solution suffers from significant shortcomings. On the one hand, it is infeasible to equip every station with a GSM/UMTS connection in the case of vast measuring networks because of the associated cost of this operation, while the provider may charge for every additional SIM card. On the other hand, there exist important locations from an environmental perspective that have poor or non-existent coverage (e.g., highly elevated regions in Swiss Alps). These drawbacks force us to search for another scalable transport technology, which may grow to reach large proportions and provide us with good coverage over remote locations. Because of recent progress in the domain of low power wireless devices we may operate Wireless Mesh Networks that allow us to significantly cut operational expenses.

Wireless Mesh Networking is an interesting communication scheme which can provide cheap Internet connectivity delivered to end users at the last mile, an easily deployable multi-hop wireless bridge between distant bases in no-direct line of sight scenarios, or a wireless back-haul connecting sensors of different purposes such as environmental monitoring or smart-home applications. To properly deploy a wireless network, there are numerous hardware and software challenges. The hardware has to be properly selected to operate under a specific power consumption regime [1], e.g., when a node is solar powered, it has to harvest and store enough energy during the day-light operation to work uninterruptedly at night. Wireless cards and antennas have to provide an acceptable signal strength to allow for high throughput, while the node setup has to provide satisfactory performance such as computational power for ciphering and packet forwarding or other network adapters able to accommodate traffic coming from wireless interfaces. The experience obtained from pilot projects installed in remote and mountainous regions for environmental monitoring [2,3] and backup backbones in urban areas illustrates that mesh networks perfectly integrate into the existing AAA (Authentication, Authorization, Accounting) [4,5], monitoring and cloud infrastructure schemes of Swiss universities. For the purpose of this work, we use Wi-Fi based backhauls to transport information from environmental sensors to Internet storage facilities in real time.

\subsection{Data Storage and Monitoring}

In addition to provisioning the transmission infrastructure, facilities for data storage and processing have to be developed. Our studies reveal several similarities between environmental monitoring in the wireless mesh setup and network monitoring provided by typical monitoring agents such as Nagios, Zabbix, and 
SNMP. In all these cases, current peripherals' status is reported to central storage for future analysis and visual presentation, while the information retrieval is triggered on a time basis. Our experience shows that we can re-use network monitoring for environmental purposes by configuring monitoring agents to constantly read out values provided by a sensor, e.g., every 15 mins. However, this methodology requires specific counter measures against Wi-Fi backhaul failures as we cannot afford the loss of environmental data if the network is inaccessible at a given time.

\subsection{Going to the Clouds}

The data collected can be processed in numerical models. In our case, we are simulating the use of groundwater water resources in a highly dynamic riveraquifer system - the Emmental ${ }^{3}$ in Switzerland. The purpose of the simulation approach is to provide a quantitative basis for sustainable water resource management. Groundwater in the aquifer of the Emmental is pumped to supply the city of Bern with drinking water. However, the abstraction of groundwater ${ }^{4}$ causes the water table in the aquifer to drop and can increase the infiltration from the river with adverse impacts on the stream ecosystems, but to what extent and how fast groundwater abstraction influences the flow in the river depends on the system state ${ }^{5}$ (i.e., how much water is stored in the aquifer). To optimize the amount of water pumped (pumping scheme), predictions on how groundwater abstraction will affect the system are required. This paper describes recent developments in data acquisition and transmission infrastructures, integrated in the data assimilation system with the goal of generating a real-time pumping scheme for the Emmental. As discussed in the next section, predictions are generated using computationally expensive models that simulate the interactions and feedback mechanisms between the river, the aquifer, the pump, and climatic forcing functions such as precipitation. The models are continually updated with acquired field data. The data assimilation approach implemented for this task requires that multiple models are run in parallel. This allows us to assess the reliability of the proposed pumping rates in a stochastic way. However, computational costs for such an approach are significant. Running a few models in parallel is challenging since even a single one may require a few days of computations on an ordinary desktop machine. We decided to use a recently developed cloud computing paradigm for our computations to speed up the whole process, while the cloud allows us to run a few parallel computing workers. Cloud computing is a growing business with many established players such as Salesforce, Amazon, Akamai, and Google. We integrated our work with the ongoing SwissACC project [6], which aims to establish a Swiss nationwide cloud computing infrastructure. Cloud providers offer several service models,

\footnotetext{
3 in German, the Emmental means the valley of the Emme river

4 i.e., pumping groundwater from the aquifer

${ }^{5}$ this system state should be understood in the physical sense, i.e., similar to states in thermodynamics and definitely not as stored information in automata theory
} 
however, we concentrate on Infrastructure as a Service (IaaS), which provides us with the required number of powerful Virtual Machines (VMs) on-demand and remote control through the Internet. The VMs are extremely useful, because they can accommodate any generic type of computations, while they do not actually require any physical maintenance from the user side. SwissACC builds upon the open-source infrastructure, OpenStack ${ }^{6}$. OpenStack comes along with the Nova controller, which automates pool managing of worker resources. For storing data in the cloud, SwissACC integrates the S3 (Simple Storage Service) driver provided by Amazon ${ }^{7}$ with the necessary API.

This work is organized in the following way. Section 2 provides a detailed problem description. In Section 3, we specify our proposed solution and we provide the most important implementation details. The results are gathered in Section 4. Finally, we conclude in Section 5.

\section{Use case: Groundwater pumping}

Drinking water supply in Switzerland is largely based on groundwater (about $80 \%$ ). Numerous water supply systems abstract groundwater close to rivers. Surface water and groundwater systems interact in highly dynamic and complex ways $[7,8]$, and therefore abstracting groundwater in the vicinity of rivers can substantially influence these dynamics [9]. Environmental laws demanding minimum water levels are in place in Switzerland. This gives rise to a challenging optimization problem. The critical parameters are the discharge in a river $^{8}$ (which is minimal due to strict environmental laws), the amount of water stored in the aquifer and the drinking water requirements. Balancing these target functions by adjusting the pumping rate thus requires a solid and quantitative understanding of the dynamics and the interactions of the river-aquifer system.

The Emmental is a perfect example that illustrates the tradeoff between the need for drinking water supply and minimal discharge in the river. The Emmental is a pre-alpine river catchment (about $200 \mathrm{~km}^{2}$ ) in central Switzerland (Fig. 1). The catchment features steep hydraulic gradients with rapid groundwater flow rates (up to $100 \mathrm{~m} / \mathrm{d}$ ). The Emme River itself is highly dynamic (discharge between 0 and $300\left(\mathrm{~m}^{3} / \mathrm{s}\right)$. The aquifer pumped close to Emme provides roughly $45 \%$ of the drinking water for Bern, the Swiss capital. Groundwater abstraction in the Emmental increases the infiltration from the river to the aquifer. In fact, during low flow periods, groundwater abstraction often causes the river to dry up. The stream water levels in the the upper Emmental are strongly affected by seasonality and are highly sensitive to dry periods. In 2003 and 2011 large stretches of the river ran completely dry, as illustrated in Fig. 2. This pronounced seasonality adds an additional level of complexity to the system.

The efficiency and sustainability of water resources management in the Emmental is directly linked to the amount of water pumped from the aquifer. A

\footnotetext{
${ }^{6}$ there obviously exist other open-source infrastructures such as OpenNebula

7 http://aws.amazon.com/s3/

${ }^{8}$ the discharge in a river is the volumetric flow rate
} 


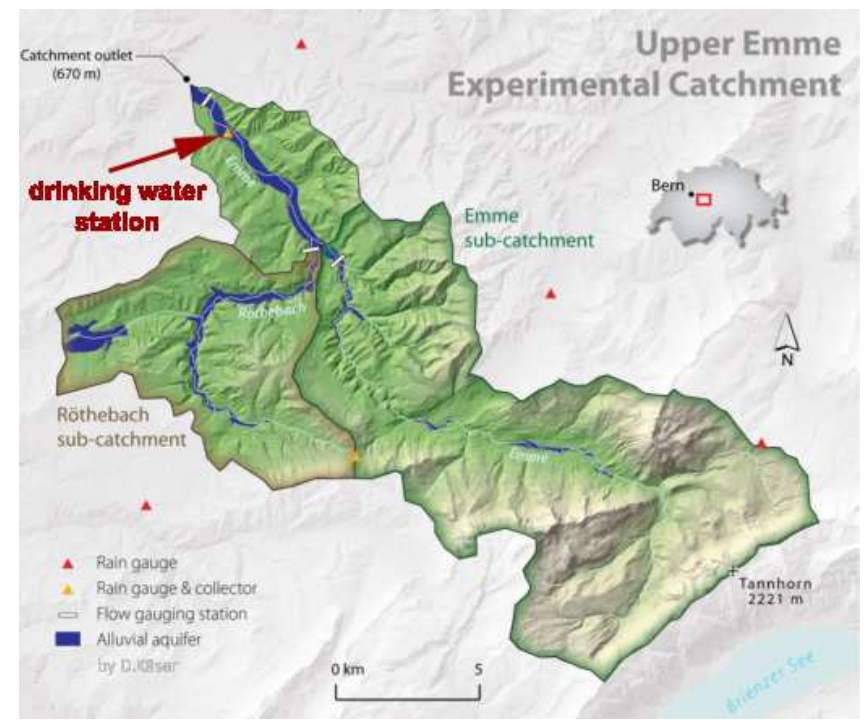

Fig. 1: The upper Emmental catchment is located close to the Swiss capital Bern (shown on the map in the top right corner). The Emme and the Roethebach rivers flow downhill from southeast and continue through the main upper Emmental after their confluence. Figure provided by D. Käser.
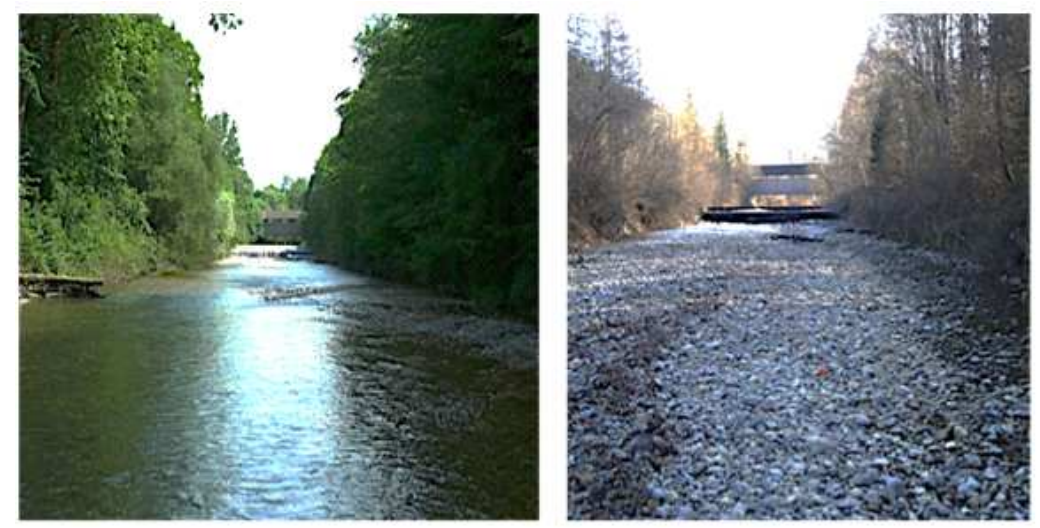

Fig. 2: A comparison between a high and a low water level situation in the upper Emmental, right below the pumping station. Photos provided by D. Käser.

quantitative approach to simulate the system is required to optimize pumping rates in this dynamic environment. Numerical models are therefore necessary for this task. By continuously incorporating field observations in the model simulation, any potential model biases can be identified, quantified and corrected. This process is called data assimilation [10]. While data-assimilation approaches are widely used in climatological models, they are rarely applied to hydrogeological 
simulations [11]. The "ingredients" for data assimilation systems are a measurement and communication network that provides field observations in real time; a data storage infrastructure; and numerical models that predict for example how different groundwater abstraction schemes affect the flow of the river. Based on these simulations, the pumping rates can be regulated in an optimal way.

\section{Implementation}

In the Emme river valley, we have established a wireless setup which contains a few stations with environmental sensors attached through USB (such as temperature and pressure meters) and other necessary stations acting as wireless backhaul thus forwarding packets and providing Internet connection (Fig. 3). From the hardware perspective, every node uses the Alix3d2 motherboards with two on-board mini-pci slots. We use the mini-pci bus to install the Winstron DNMA-92 IEEE 802.11abgn interfaces, while our wireless links are provided by directional antennas of high-gain. When the electric power grid is not available, we equip a node with a solar panel and battery to secure a continuous 24 hours operation (normally, the battery charges during the day-light operation). Our nodes are placed in a special-purpose enclosure which protects them against outdoor conditions, e.g., humidity. When a high number of Wi-Fi interfaces are required, we gather a few mother boards together in a single box. The Linux based ADAM system ${ }^{9}$ serves as the operating system platform. Due to the installed OLSR and IEEE 802.11s, the network is easily expandable, i.e., the installation of a new node requires little attention from the administration perspective.

\subsection{Environmental Monitoring}

Zabbix ${ }^{10}$ provides a client-server infrastructure which allows us to monitor and control remote machines. There are a large number of predefined parameters, while Zabbix also provides an opportunity to launch user-defined commands to support user-specific peripherals. Due to this feature, we are able to equip Zabbix agents with drivers, i.e., special purpose applications which read out environmental parameters from the sensors through USB and provide the agent with the received data. We deployed one running instance of the Zabbix agent on every node in the mesh and one instance of the Zabbix server at the central storage (online database). To control the Zabbix server (e.g., including another sensor), we are provided with an advanced back-end web interface and rich logging system.

The online database allows the access of the measured environmental system state in real-time, providing the basis for a real-time forecasting system to control the groundwater abstraction rates. Periodically, our software asks sensors about

\footnotetext{
${ }^{9}$ developed by the University of Bern: http://cds.unibe.ch

${ }^{10}$ http://www.zabbix.org
} 


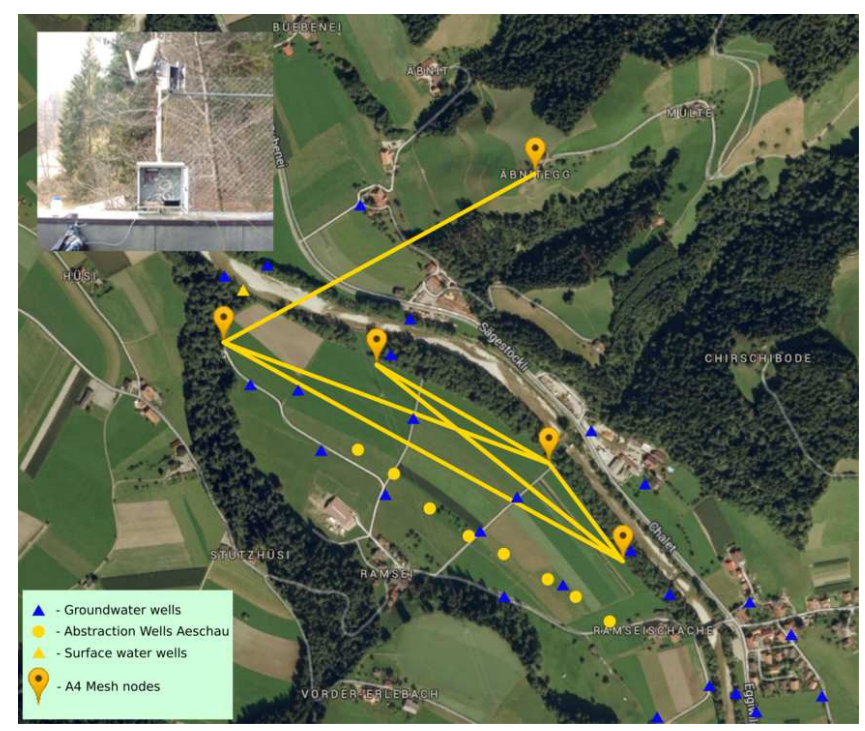

Fig. 3: Environmental setup of the network

current values of the measurements. Then, again periodically, the Zabbix server executes a remote driver on the nodes and obtains values of the measurements. This data is transferred to the database on the server. Finally, the data can be accessed through the web interface (Fig. 4). The Zabbix infrastructure provides us with wide variety of tools for drawing plots and applying simple formulas to the data; it fits well to the requirements of our application context.

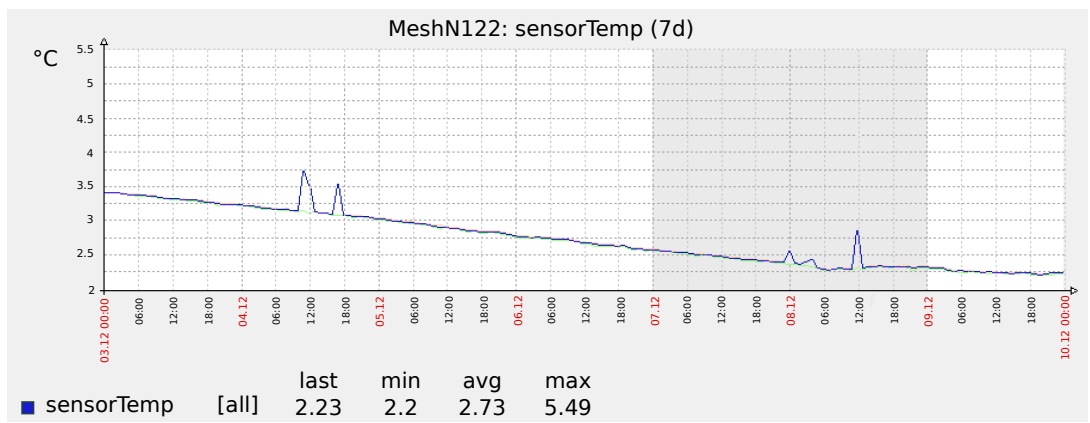

Fig. 4: Online environmental data output (the larger peaks appear to be artefacts) 


\section{$3.2 \quad$ Real-time modeling}

The latest generation of numerical models is now able to simulate the interactions between surface water and groundwater in a fully coupled way [12]. One of the most advanced codes in this respect is HydroGeoSphere [13]. In addition to simulating surface water and groundwater interactions, the code can also simulate vegetation dynamics as well as the recharge ${ }^{11}$ and discharge processes in response to precipitation, evapotranspiration or groundwater abstraction. HydroGeoSphere is therefore used to simulate the Emmental system.

The geometric setup is based on a high resolution digital terrain model. The numerical coupling between the surface and subsurface domain is conceptualized through a dual node approach, as described in [13]. The model requires a very large amount of parameters, such as hydraulic properties of the streambed, the soil or the aquifer. These parameters cannot be measured in the field at the required spatial resolution and therefore have to be estimated. Numerous approaches are available in this regard. A "classic" way is to adjust parameters in order to minimize the mismatch between the available historical measurement data and the corresponding model simulations. Once a model reproduces historic measurement data satisfactorily, it is used to predict future system states under changing forcing functions. However, all numerical models are a simplification of reality, both in terms of the processes considered as well as in their parameterization. Therefore, any calibrated model will sooner or later deviate from the real, physical system state. Clearly, the model state (i.e., the simulated water levels or the actual discharge in the river) has to be as close as possible to the real system in order to provide reliable predictions on how a planned pumping scheme will affect the system in the near future. Therefore, the "classical" calibration approach is not well suited for this application. By using a data assimilation approach, the model is continuously updated in terms of its state and parameters. We implement a data assimilation approach similar to the work of [11]. Currently, the assimilated measurements consist of stream level and water tables along the river.

HydroGeoSphere is a numerically demanding code, and the highly dynamic interactions between surface water and ground water require a fine temporal discretization scheme. Moreover, numerous models are running in parallel to explore the influence of different pumping schemes, as well as different possible model parameterizations. To accomplish this computational burdon, significant computational resources are required (see next section). The multiple simulations of possible abstraction rates with the corresponding predicted impacts on the river-aquifer system allow us to identify the optimal pumping volumes in consideration of the environmental laws and drinking water demands. With the development of this simulation system, the pump can be operated in an optimal way. However, a remote regulation system that transmits the optimal pumping rates to the pump must be implemented.

\footnotetext{
$\overline{11}$ water infiltrating the soil reaching the underground water table
} 


\subsection{Swiss Academic Compute Cloud}

Our Cloud based solution allows researchers to perform resource consuming computations with minimal efforts. Firstly, the data collected with the environmental sensors is stored on the pilot-project Swiss Academic Compute Cloud (SACC), a unified cloud service providing storage and computation resources for Swiss academic institutes, which makes use of a specialized S3-based cloud repository - the Object Storage (OS). The user front-end is developed in Django ${ }^{12}$, which is a free open source Python based web application framework that provides us with the model-view-controller architectural paradigm. Our currently implemented frontend allows a researcher to visit the web-page, choose required input data, initiate required tasks, and download the results of completed computations. Behind the user front-end, we integrate a Python engine - GC3Pie ${ }^{13}$, which is developed by the GC3 group at the University of Zürich ${ }^{14}$ and enables all cloud related operations such as starting and stopping new workers. We configured our framework to run several instances of HydroGeoSphere on allocated working VMs. Due to the infrastructure configuration, every instance of the HydroGeoSphere is provided with the input files from the OS, which in turn also acts as a storage facility for models returned by completed instances. This OS-based data organization scheme is important, because it provides portability as there are many different cloud providers supporting this storage manner.

\section{Results}

Firstly, we deployed a measuring and transporting mesh network in the Emmental which proves its high performance and reliability in harvesting environmental data. Secondly, the first numerical HydroGeoSphere model that is capable of simulating the interactions and feedback mechanisms between the river, the aquifer and the pumps has been set up. It includes the integration of the HydroGeoSphere binary with the cloud computing workers, implementing the web-interface for running tasks, and integrating the OS for maintaining both the input and output of the HydroGeoSphere. All parts of the so far implemented infrastructure fully correspond to our requirements. Due to the integration with cloud infrastructures, simultaneous running of different models showed us significant profit in comparison with the usual sequential running. Also, the web interface for controlling the computations, greatly simplified the whole process of launching models.

The current infrastructure is under ongoing developments. In the future, we plan to strongly integrate all the technological pieces to allow for fully automated model computations thus providing valuable pumping scheme predictions in real time. We also plan to develop our web interface to allow for any generic computational use-case. One of the identified improvements relates to precise definition

\footnotetext{
12 http://www.djangoproject.com

13 http://code.google.com/p/gc3pie/

14 www.gc3.uzh.ch
} 
of input and output to support many different applications (e.g., by employing XML to define program options, input/output files, etc.).

\section{Conclusions}

The integration of advanced Information Technologies in environmental simulation systems allows for a new dimension of natural resource management. Our proposed solution is especially interesting for remote locations with harsh environmental conditions in which wireless mesh network prove to provide a reliable network infrastructure. When the transporting infrastructure is developed, one can employ cloud computing to solve any computationally expensive problem, while the network monitoring application (e.g., Zabbix) can transport information in different use-cases such as environmental monitoring or smart-home applications.

\section{Acknowledgements}

This work is partially funded by the Swiss State Secretariat for Education and Research through SWITCH and the Swiss National Science Foundation through NRP 61 on Sustainable Water Management. We particularly thank Torsten Braun (the leader of the A4Mesh project) and his team as well as Sergio Mafioletti (the leader of the SwissACC project) for their contributions to this work.

\section{References}

1. Badawy, G., Sayegh, A., Todd, T.: Solar powered wlan mesh network provisioning for temporary deployments. In: Wireless Communications and Networking Conference, 2008. WCNC 2008. IEEE. (March 2008) 2271-2276

2. Wu, D., Mohapatra, P.: Qurinet: A wide-area wireless mesh testbed for research and experimental evaluations. In: Communication Systems and Networks (COMSNETS), 2010 Second International Conference on. (Jan 2010) 1-10

3. Jamakovic, A., Dimitrova, D., Anwander, M., Macicas, T., Braun, T., Schwanbeck, J., Staub, T., Nyffenegger, B.: Real-world energy measurements of a wireless mesh network. In Pierson, J.M., Da Costa, G., Dittmann, L., eds.: Energy Efficiency in Large Scale Distributed Systems. Lecture Notes in Computer Science. Springer Berlin Heidelberg (2013) 218-232

4. Anwander, M., Braun, T., Jamakovic, A., Staub, T.: Authentication and authorisation mechanisms in support of secure access to wmn resources. In: World of Wireless, Mobile and Multimedia Networks (WoWMoM), 2012 IEEE International Symposium on a. (June 2012) 1-6

5. Schiller, E., Monakhov, A., Kropf, P.: Shibboleth based authentication, authorization, accounting and auditing in wireless mesh networks. In: LCN. (2011) 918-926

6. Kunszt, P., Maffioletti, S., Flanders, D., Eurich, M., Schiller, E., Bohnert, T., Edmonds, A., Stockinger, H., Jamakovic-Kapic, A., Haug, S., Flury, P., Leinen, S.: Towards a swiss national research infrastructure. In: Proceedings of the 1st international workshop on federative and interoperable cloud infrastructures 2013, FedICI13 organized in conjunction with Euro-par 2013. (aug 2013) 
7. Partington, D., Brunner, P., Frei, S., Simmons, C.T., Werner, A.D., Therrien, R., Maier, H.R., Dandy, G.C., Fleckenstein, J.H.: Interpreting streamflow generation mechanisms from integrated surface-subsurface flow models of a riparian wetland and catchment. Water Resources Research 49(9) (2013) 5501-5519

8. Brunner, P., Cook, P.G., Simmons, C.T.: Disconnected surface water and groundwater: from theory to practice. Ground Water 49(4) (2011) 460-7

9. Winter, T.C., Harvey, J.W., Franke, O.L., ALLEY, W.M.: Ground Water and Surface Water A Single Resource. USGS, Circular 1139. Denver, Colorado. (1998)

10. Evensen, G.: Data assimilation : the ensemble Kalman filter. 2nd edn. Springer, Dordrecht ; New York (2009)

11. Hendricks Franssen, H.J., Kinzelbach, W.: Ensemble kalman filtering versus sequential self-calibration for inverse modelling of dynamic groundwater flow systems. Journal of Hydrology 365(3-4) (2009) 261-274

12. Brunner, P., Simmons, C.T.: Hydrogeosphere: A fully integrated, physically based hydrological model. Ground Water 50(2) (2012) 170-176

13. Therrien, R., McLaren, R., Sudicky, E., Panday, S.: HydroGeoSphere. Groundwater Simulations Group (2013) 